\title{
Genetic analysis of grain weight in wheat
}

\author{
A. J. S. Chojecki,* \\ M. W. Bayliss $\dagger^{*}$ \\ and M. D. Gále
}

\author{
Plant Breeding Institute, Maris Lane, Trumpington, \\ Cambridge CB2 2LQ, U.K. \\ $\dagger$ Imperial Chemical Industries PLC, The Heath, \\ Runcorn, Cheshire, U.K.
}

The reciprocal monosomic analysis (RMA) technique of intervarietal chromosome manipulation was used to investigate the genetic control of grain weight in two varieties of wheat (Spica and Chinese Spring). The method enabled major genetic differences between the two varieties for this quantitative character to be assigned to individual chromosomes.

The RMA was extended by the extraction of reciprocal disomic $F_{3}$ families, of which individual ears were analysed at maturity for grain weight at each position within the ear. Chromosomes $1 \mathrm{~A}, 1 \mathrm{D}$ and $7 \mathrm{~A}$ of the large-grained parent, Spica, increased grain weight relative to the homologous chromosomes of the small-grained parent, Chinese Spring. The effect of chromosomes $1 \mathrm{D}$ and $7 \mathrm{~A}$ were proportionately similar throughout the ear, while that of $1 \mathrm{~A}$ was disproportionately greater in the distal florets of each spikelet. None of these differences involved simultaneous effects on grain number and thus could be considered to be specific genetic effects on grain growth.

\section{INTRODUCTION}

A conventional biometrical analysis of the genetic differences between two varieties of wheat (Triticum aestivum, $2 n=6 x=42$ ) is often unsuitable for the identification of genes with major effects on quantitative characters because many genes are likely to be involved and their individual effects cannot be distinguished. Furthermore, in the case of grain weight at maturity, it is usually not possible to separate competitive effects on grain weight due to differences in grain number per ear from direct effects on grain development. Even varieties having the same mean number of grains per ear may have different numbers of spikelets and grain-bearing florets per spikelet, which can confuse the interpretation of genetic differences in grain weight.

However, methods of genetic analysis involving intervarietal chromosome manipulation can be used to separate the effects of each chromosome of the genome so that the major chromosomal effects on a particular character can be identified. These methods range from the relatively simple, such as conventional $F_{1}$ or $F_{2}$ monosomic analysis (Sears, 1953; Kuspira and Unrau, 1959), to the

\footnotetext{
* Present address: I.C.I. International Seeds Business, Jealott's
} Hill Research Station, Bracknell, Berks. sophisticated but labour-intensive, such as development and analysis of intervarietal chromosome substitution lines (Kuspira and Unrau, 1957; Law, 1967). The choice of method depends on the nature of the character being studied (qualitative or quantitative), the availability of appropriate aneuploid lines and the time and effort that it is practical to spend on the analysis.

The method used here was that of reciprocal monosomic analysis, first described and used by McEwan and Kaltsikes (1970) and subsequently by Law et al. (1979; 1981). It enables the detection and chromosomal location of allelic differences between two varieties by comparing the effects of each chromosome in one variety with those of its reciprocal homologous chromosome in the other variety. In addition to the identification of large genetic effects on grain weight, it was possible to overcome the problems of interpretation caused by the confounding effects of grain number changes, by producing lines differing in grain weight, but identical in terms of grain number and ear-structure.

\section{MATERIALS AND METHODS}

Reciprocal monosomic analysis (RMA) involves reciprocal crosses between homologous monosomics of two varieties, to exploit the differential 
transmission of the hemizygous chromosome through the pollen and the egg (Sears, 1953). For each chromosome, this results in a pair of reciprocal monosomic $F_{1}$ hybrids which differ in the unrecombined hemizygous chromosome but have identical heterozygous genetic backgrounds (fig. 1). The complete analysis involves the production of 21 reciprocal pairs. Phenotypic differences between individuals of a pair are due to allelic differences in genes carried on the hemizygous chromosomes. It is thus possible to assess the additive genetic contribution of each chromosome to the parental difference.

The parent varieties used here were Chinese Spring (CS) and Spica. Monosomic sets exist for both varieties, that of CS was produced by Professor E. R. Sears, University of Missouri, Columbia, U.S.A. and the Spica monosomics were developed and kindly provided by
Dr R. A. McIntosh, Plant Breeding Institute, University of Sydney, Australia.

For each monosomic line of Spica and CS at least four 41-chromosome individuals were identified cytologically by counting chromosomes in dividing root tip cells. The reciprocal hybridisations were made on a "plant-to-plant" basis. That is, one ear of a Spica monosomic plant provided pollen to apply to an emasculated ear of a CS plant monosomic for the same chromosome, and, for the reciprocal cross, a different ear of the same Spica monosomic plant was used as the female parent to be pollinated by the same CS monosomic plant. For each reciprocal line, four 41-chromosome $F_{1}$ plants were grown in separate $16 \mathrm{~cm}$ pots in a randomised block design on the glasshouse bench. To provide additional uniformity, each plant was restricted to the mainstem plus three tillers, by removing extra tillers as they developed.

RECIPROCAL MONOSOMIC ANALYSIS

PARENTS

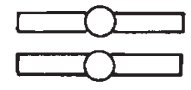

CS MONO

$(2 n=41)$

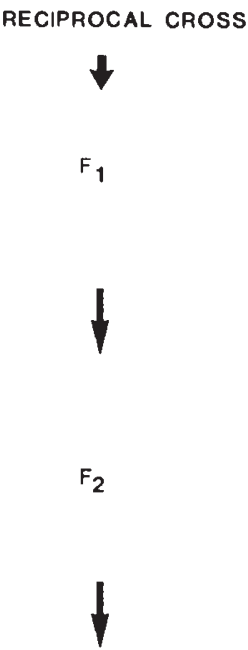

$\mathrm{F}_{3}$
CS MONO $\&$ × $\sigma^{\pi}$ SPICA MONO
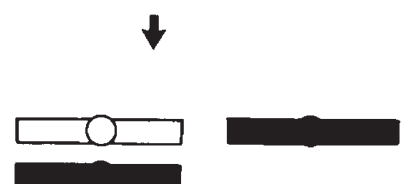

IDENTIFY MONOSOMIC $F_{1} s$
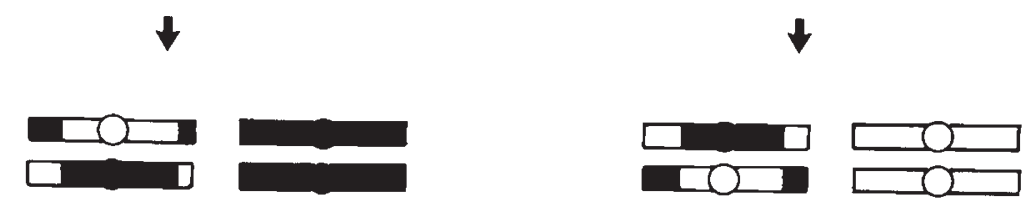

IDENTIFY DISOMIC $\mathrm{F}_{2} S \quad(2 n=42)$ AND SELF

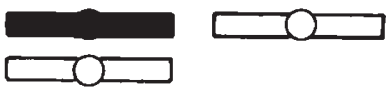

SPICA MONO \& $x$ O CS MONO
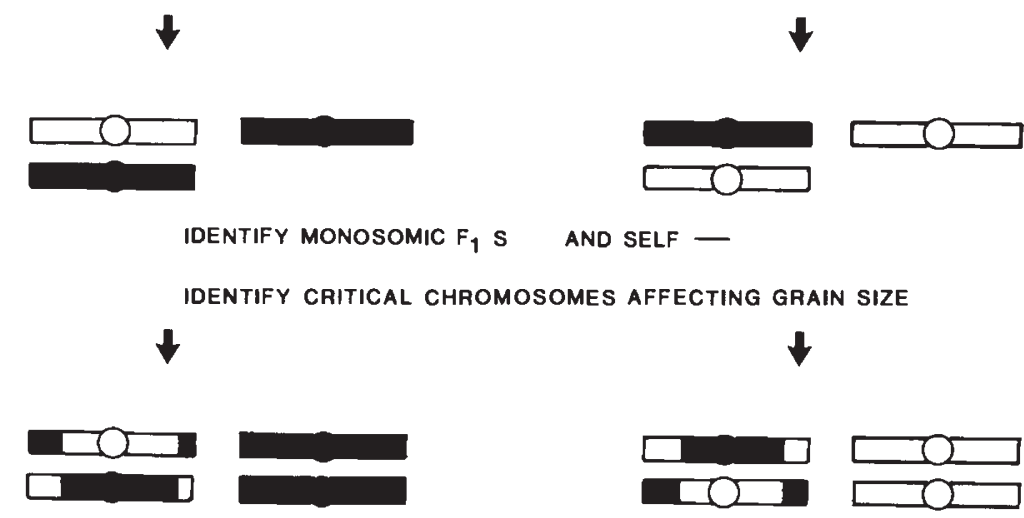

IDENTIFY CRITICAL CHROMOSOMES AFFECTING GRAIN SIZE

$(2 n=41)$
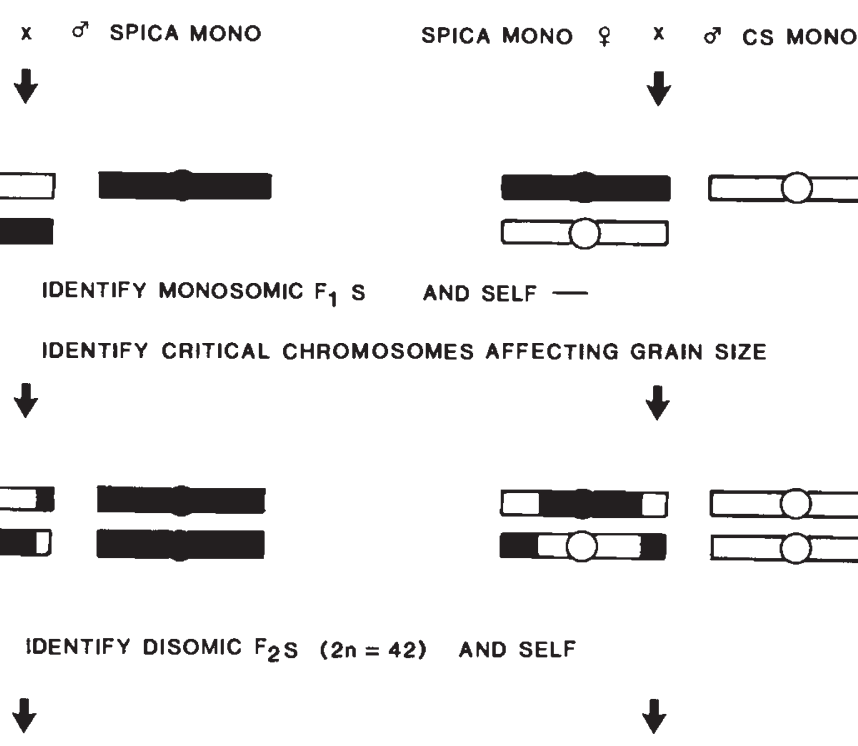

ANALYSIS OF GRAIN SIZE OF CRITICAL TRUE BREEDING DISOMIC RECIPROCAL SUBSTITUTIONS

IN IDENTICAL SEGREGATING GENETIC BACKGROUNDS

Figure 1 The reciprocal monosomic $F_{1}$ method of analysis, with subsequent extraction of disomic lines. 
Yield component analysis was carried out on these $F_{1}$ individuals to identify the critical chromosomal effects for the extended $F_{2}$ analysis. For the five chosen chromosomes the RMA method was extended by the extraction of disomics (fig. 1). Grains from the selfed $F_{1}$ plants were germinated and six 42-chromosome individuals were identified for each reciprocal. These euploid $F_{2}$ lines were then selfed to produce reciprocal disomic $F_{3}$ families, each consisting of six lines. The background genotype varies between lines but the average background effect of each reciprocal set of six lines is likely to be similar. Differences between the means of the two families of a reciprocal pair are thus due to allelic differences in genes carried on the unrecombined, homozygous pair of chromosomes.

The parental euploids and reciprocal disomic $\mathrm{F}_{3}$ families were sown in the field as spaced plants in two randomised blocks, surrounded by guard plots. Each $\mathrm{F}_{3}$ line was represented by one plot in each of the randomised blocks. Each plot consisted of a row of 11 plants at $10 \mathrm{~cm}$ spacings, with $30 \mathrm{~cm}$ between rows. Six rows each of CS and Spica were grown in each block. Mainstem ears from three central plants within each plot were harvested at maturity.

A profile of grain weight throughout the ear was obtained by weighing each grain in the ear individually. The data concerning grain weight and relative position in the ear was automatically transferred to, and processed on, a microcomputer. To permit comparisons to be made between ears of differing structure (see fig. 3), the data were expressed for each floret level (up to six) over three main regions or spikelets (plus four extreme spikelets).

\section{RESULTS}

\section{(i) Reciprocal monosomic $F_{1}$ analysis of grain weight and grain number (Glasshouse experiment)}

Spica had a final mean grain weight of $54.3 \mathrm{mg}$ compared with $32.6 \mathrm{mg}$ for CS (fig. 2). This difference in grain weight was offset by the difference in mean grain number per ear $(28 \cdot 1$ for Spica, $44 \cdot 1$ for CS). As a result the mean grain yield per ear did not differ between Spica and CS. The $\mathrm{CS} \times$ Spica and Spica $\times$ CS reciprocal euploid hybrids both had the same mean grain weight and grain number per ear, intermediate to the two parents. This indicates that there are no maternally (cytoplasmically) inherited factors influencing grain weight or grain number, in CS and Spica.

Among the reciprocal monosomic $F_{1}$ hybrids for chromosomes $1 \mathrm{~A}, 1 \mathrm{D}, 3 \mathrm{~A}, 4 \mathrm{~B}, 5 \mathrm{~B}, 7 \mathrm{~A}, 7 \mathrm{~B}$ and $7 \mathrm{D}$ the reciprocal containing the hemizygous Spica homologue had a greater mean grain weight than that carrying the hemizygous CS homologue. No CS chromosome produced a significant increase in grain weight relative to its Spica homologue.

Spica chromosomes 2B and 6A increased grain number per ear, and $3 \mathrm{D}$ and $7 \mathrm{~B}$ depressed it, relative to their respective $\mathrm{CS}$ homologues. In general, the fertility of the $F_{1}$ monosomic lines was lower than that of the euploid $F_{1}$. Some of the most marked reductions in fertility were associated with monosomic hybrids involving group 2 chromosomes. Reduced fertility is characteristic of monosomics of this group (Sears, 1954).

Only chromosomes that affected grain weight in the absence of potentially compensatory effects on grain number could be considered to be unequivocally responsible for direct control of grain weight. Spica chromosomes 1A, 1D, 3A, 4B and $7 \mathrm{~A}$ each increased grain weight relative to their CS homologue and did not affect grain number per ear. Spica chromosomes 5B and 7D also increased grain weight but showed non-significant indications of a depressive effect on grain number. Thus, chromosomes 1A, 1D, 3A, 4B and 7A were chosen for extraction of reciprocal disomic lines.

\section{(ii) Reciprocal disomic $F_{3}$ analysis of grain weight and grain number (Field experiment)}

As in the glasshouse experiment, Spica had larger grains than CS (table 1), again offset by a lower grain number per ear, resulting in a small increase in net grain yield.

In the comparison of reciprocal disomic $F_{3}$ families, chromosomes 1A, 1D and 7A of Spica increased mean grain weight by 5.3 to $5.9 \mathrm{mg}$ rela. tive to the families containing the respective disomic CS homologues (table 1). As in the $F_{1}$ analysis these chromosomes had no effect on grain number per ear. As a result Spica chromosomes 1D and $7 \mathrm{~A}$ were associated with a significant increase in mean grain yield per ear, relative to the CS homologues. Spica $1 \mathrm{~A}$ produced a non-significant increase in grain yield of 11 per cent. Chromosomes $3 \mathrm{~A}$ and $4 \mathrm{~B}$ affected neither mean grain weight nor grain number per ear.

The profile of grain weight and number is illustrated in fig. 3 which shows diagramatically for CS and Spica the mean weight per grain in grainbearing florets and the actual mean yield per floret, 


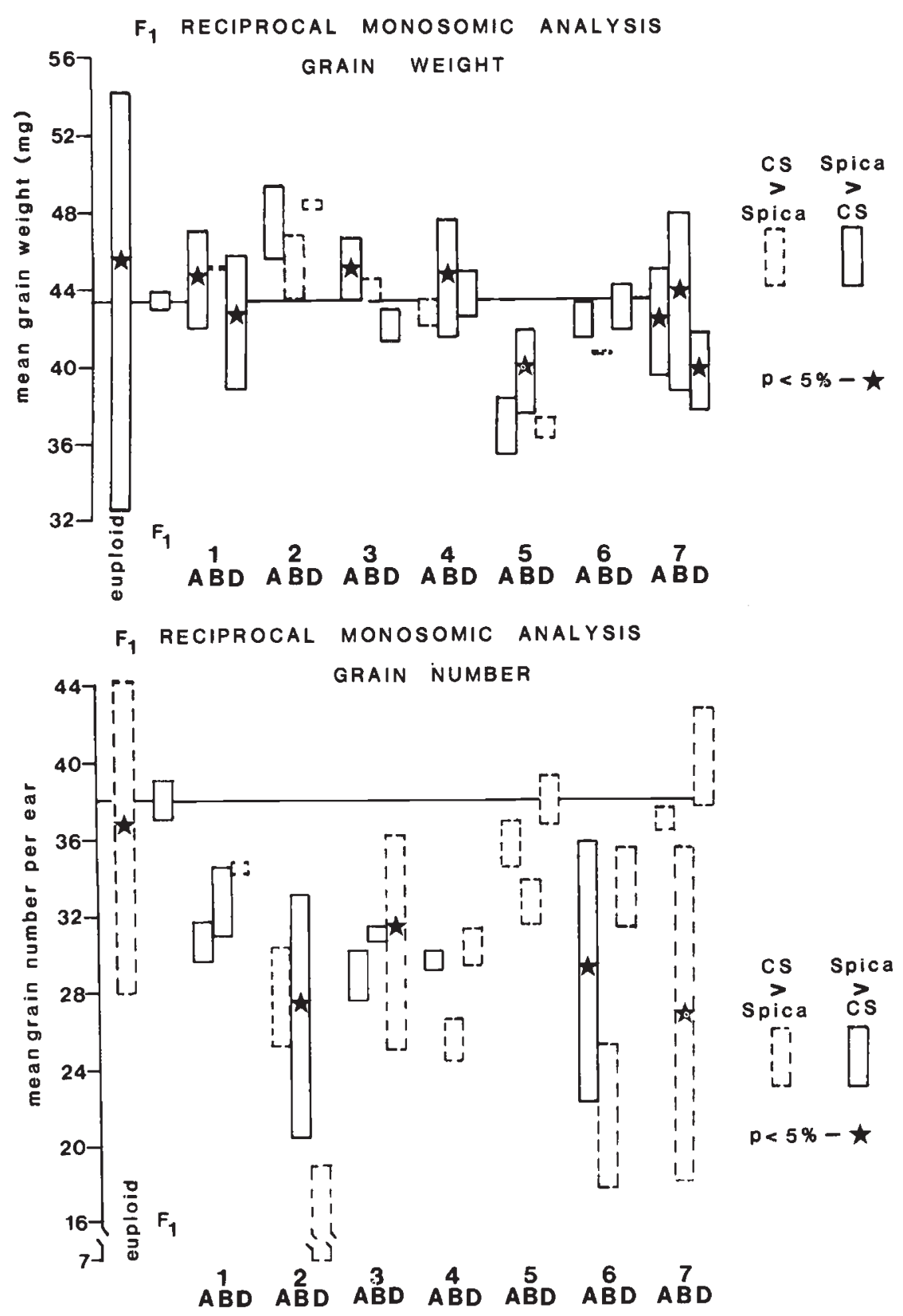

Figure 2 (a) Grain weight and (b) grain number per ear on $F_{1}$ reciprocal monosomic plants from the Chinese Spring $\times$ Spica analysis. Note: Each bar represents the reciprocal difference between the $F_{1}$ monosomic hybrids containing hemizygous Spica and CS homologues. An unbroken line indicates that the hybrid containing the Spica homologue has greater grain size or number than that containing CS homologue. A broken line indicates that the hybrid containing the CS homologue is greater than that containing Spica homologue. The horizontal line represents the euploid $F_{1}$ mean. LSD $(5$ per cent $)=3 \cdot 53 \mathrm{mg}, 6 \cdot 41 \mathrm{grains}$.

taking empty florets into account. Although Spica had larger grains at every position, CS had more grain-bearing florets in each spikelet and more spikelets per ear. In both varieties there was a progressive decline in grain weight in distal florets. Also, the weight and number of grains was lower in the third and apical spikelet divisions than in divisions 1 and 2, and this effect was most marked in more distal florets.

In the ear profile analysis there were no reciprocal differences between $\mathrm{F}_{3}$ disomic families in terms of the pattern of grain-bearing florets in 
each spikelet. The effect of chromosomes 1D and $7 \mathrm{~A}$ on mean weight per grain was due to an effect on grain weight that was proportionately similar at each grain-bearing floret. In contrast, however, the effect of chromosome 1A on grain weight was disproportionately greater in florets 2 and 3 than in other florets (table 2) and this effect was in evidence in each spikelet division.

Chromosomes $3 \mathrm{~A}$ and $4 \mathrm{~B}$ were not associated with any effects on the profile of grain weight or number throughout the ear. Differences between $\mathrm{CS}$ and Spica in the hemizygous effectiveness of alleles on chromosomes $3 \mathrm{~A}$ and $4 \mathrm{~B}$ could explain why effects detected in the $F_{1}$ monosomics were not seen in the $F_{3}$ disomics.

\section{DISCUSSION}

\section{(i) Analysis of grain weight}

Final grain weight is determined later in plant development than the other principle yield components, grain number per ear and number of ears per unit ground area (i.e., tiller number). Thus any

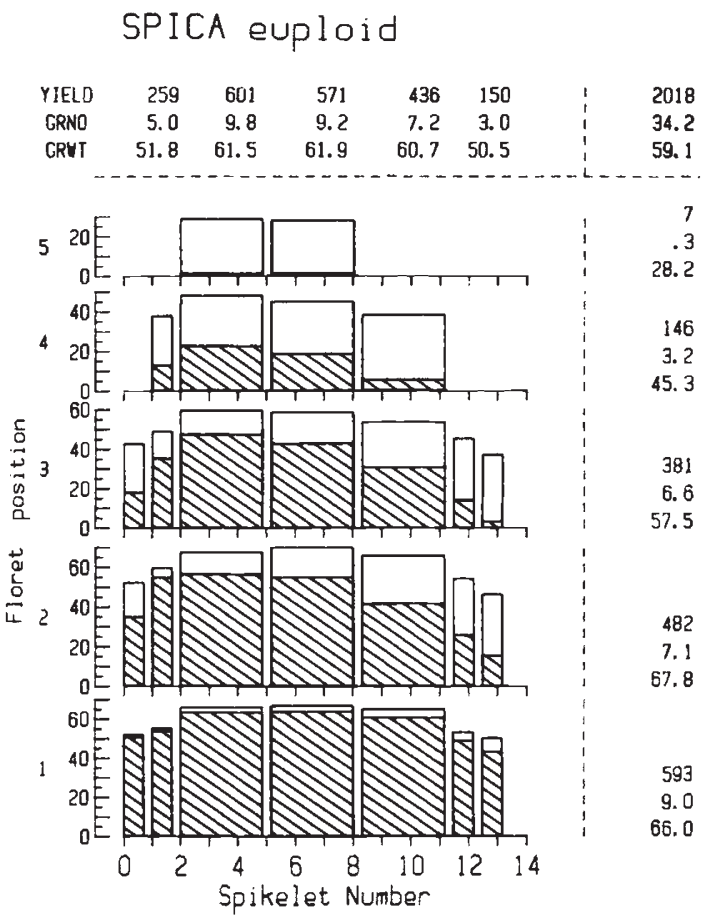

Table 1 Yield component analysis for CS, Spica and reciprocal disomic $F_{3}$ lines. Each value represents the mean of all grains from up to 36 ears. LSD's for CS and Spica were obtained from the variance between 36 ears, one from each of 36 plants. Among the pairs of reciprocal disomic $\mathrm{F}_{3}$ lines, the variances between six lines per genotype were homogeneous and were pooled to obtain the displayed LSDs

\begin{tabular}{llll}
\hline Genotype & $\begin{array}{l}\text { Mean weight } \\
\text { per grain } \\
(\mathrm{mg})\end{array}$ & $\begin{array}{l}\text { Mean grain } \\
\text { number } \\
\text { per ear }\end{array}$ & $\begin{array}{l}\text { Mean grain } \\
\text { yield per } \\
\text { ear }(\mathrm{mg})\end{array}$ \\
\hline Spica & $59 \cdot 1^{\ddagger}$ & $34 \cdot 2^{\ddagger}$ & 2018 \\
CS & $32 \cdot 8^{\ddagger}$ & $55 \cdot 9$ & 1834 \\
$\quad$ LSD 5\% & $2 \cdot 8 \mathrm{mg}$ & $5 \cdot 79$ grains & $351 \cdot 3 \mathrm{mg}$ \\
Spica 1A & $45 \cdot 7 \dagger$ & $41 \cdot 0$ & 1880 \\
CS 1A & $40 \cdot 4$ & $42 \cdot 1$ & 1681 \\
Spica 1D & $49 \cdot 9 \dagger$ & $42 \cdot 5$ & $2070 *$ \\
CS 1D & $44 \cdot 0$ & $39 \cdot 5$ & 1695 \\
Spica 3A & $48 \cdot 2$ & $44 \cdot 2$ & 2140 \\
CS 3A & $48 \cdot 5$ & $42 \cdot 2$ & 2045 \\
Spica 4B & $42 \cdot 3$ & $39 \cdot 1$ & 1660 \\
CS 4B & $44 \cdot 0$ & $39 \cdot 1$ & 1717 \\
Spica 7A & $49 \cdot 9 \dagger$ & $44 \cdot 3$ & $2214 \dagger$ \\
CS 7A & $44 \cdot 2$ & $39 \cdot 6$ & 1762 \\
$\quad$ LSD 5\% & $4 \cdot 8 \mathrm{mg}$ & $7 \cdot 0$ grains & $377 \cdot 2 \mathrm{mg}$ \\
\hline
\end{tabular}

$* P<0.05, \dagger, P<0.01, \ddagger P<0.1$.

Figure 3 Mean grain weight and yield at each floret level within the ear, Chinese Spring and Spica. Note: Each figure represents the mean of 36 ears. Two values are displayed at each floret level within each spikelet division: (a) the full height of the bar represents the mean weight per grain $(\mathrm{mg})$, disregarding sterile florets and (b) the shaded portion represents the mean yield per floret $(\mathrm{mg})$, including sterile florets. The mean weight per grain, grain number and grain yield for each floret level (excluding the two most extreme spikelets) and for each spikelet region are displayed at the top and side of each ear profile. The grand mean for these parameters is shown in the upper right-hand corner. 
Table 2 Mean grain weight and grain number within ears of reciprocal disomic families for chromosome $1 \mathrm{~A}$ of CS and Spica. For the purposes of the analysis the ear was divided into five regions of spikelets. The basal and apical divisions contained two extreme spikelets each, while the remaining spikelets were divided into three equal divisions. LSD ( 5 per cent $)=4.47 \mathrm{mg}, 1.83$ grains

\begin{tabular}{llllll}
\hline & \multicolumn{2}{c}{ Grain weight $(\mathrm{mg})$} & & \multicolumn{2}{c}{ Grain No. } \\
\cline { 2 - 3 } \cline { 5 - 6 } Floret & Spica 1A & CS 1A & & Spica 1A & CS 1A \\
\hline 1 & $51 \cdot 0$ & $47 \cdot 7$ & $9 \cdot 1$ & $8 \cdot 9$ \\
2 & $53 \cdot 2$ & $46 \cdot 4 \dagger$ & $6 \cdot 4$ & $7 \cdot 2$ \\
3 & $48 \cdot 1$ & $41 \cdot 1 \dagger$ & & $7 \cdot 5$ & $7 \cdot 2$ \\
4 & $38 \cdot 1$ & $33 \cdot 7$ & & $5 \cdot 8$ & $6 \cdot 3$ \\
5 & $31 \cdot 6$ & $26 \cdot 4$ & $2 \cdot 5$ & $2 \cdot 7$ \\
6 & $34 \cdot 0$ & $20 \cdot 2$ & $0 \cdot 2$ & $0 \cdot 5$ \\
Spikelet division & & & \\
Basal & $41 \cdot 9$ & $36 \cdot 8^{*}$ & $6 \cdot 0$ & $6 \cdot 1$ \\
1 & $47 \cdot 0$ & $41 \cdot 3^{*}$ & $11 \cdot 8$ & $12 \cdot 6$ \\
2 & $48 \cdot 2$ & $42 \cdot 4^{*}$ & $10 \cdot 8$ & $11 \cdot 4$ \\
3 & $45 \cdot 1$ & $40 \cdot 4^{*}$ & $8 \cdot 8$ & $8 \cdot 1$ \\
Apical & $40 \cdot 3$ & $35 \cdot 1^{*}$ & $3 \cdot 6$ & $3 \cdot 2$ \\
\hline
\end{tabular}

$\$$ Only florets 1-4 were included in the analysis of variance because there were very few grains set in florets 5 and 6 . LSD $(5$ per cent $)=4.81 \mathrm{mg}$ per grain, 1.27 grains. ${ }^{*} P<0.05, \dagger P<$ 0.01 .

genetical modifications or chemical treatments designed to increase yield through increases in grain weight are less likely to be offset by compensatory changes in other yield components.

Because of the interrelationship of mature grain weight and grain number per ear (e.g., Bingham, 1967; Lupton and Kirby, 1968), it is difficult to interpret differences in grain weight in the presence of differences in grain number per ear. Experiments involving artificial sterilisation of florets and grainremoval treatments have attempted to offset differences in grain number by making comparisons on uniformly trimmed ears (e.g., Brocklehurst, 1977; Bremner and Rawson, 1978; Simmons and Moss, 1978). However, such experiments may involve unsound assumptions concerning the redistribution of assimilate in surgically treated ears (Wardlaw and Moncur, 1976; Jenner, 1980).

A preferable approach is the use of chromosome manipulation, as here, to produce lines that differ in grain weight but are identical in terms of grain number per ear and floret fertility. Thus, the effects of chromosomes $1 \mathrm{~A}, 1 \mathrm{D}$ and $7 \mathrm{~A}$ on grain weight could be considered to be specifically on grain growth, rather than the result of a competitive interaction with grain number or position within the ear. Other genetic analyses involving wheat aneuploids have shown that variation in mean grain weight is controlled to a greater or lesser extent by each of the chromosomes in the genome (e.g., Law and Worland, 1973), and that the nature of the chromosomal effects depend on the parent genotypes. The effects on grain weight detected here therefore represent parts of the overall difference in grain weight between CS and Spica. In this respect, these genotypes provide a suitable experimental system in which to investigate specific aspects of grain development that control final grain weight. These developmental studies will be reported elsewhere.

The analysis of ear profiles showed that differences in mean grain weight may be achieved through general effects throughout the ear (e.g., chromosomes 1D and 7A) or through disproportionately greater effects at particular floret levels (chromosome 1A). Thus, an analysis confined for example to basal florets of central spikelets would have failed to detect the most pronounced effect of chromosome $1 \mathrm{~A}$ on grain weight, which was at florets 2 and 3. This illustrates that, because mean grain weight is derived from a population of different grains, it is necessary to analyse the pattern within this population, if an effective description of the character is to be achieved.

\section{(ii) Genetic analysis}

The use of reciprocal monosomic analysis with subsequent extraction of disomics permits the identification of chromosomes carrying genes that have large effects relative to those segregating in the genetic background. Moreover the design of the analysis allows the identification of genes which are not effective in the hemizygous state and also overcomes any confounding effects due to background genetic differences within monosomic stocks. Genes identified by RMA are likely to be those most useful for incorporation into breeding programmes. Genetic effects such as these can also be detected using intervarietal chromosome substitution line analysis, but the production of these lines requires four to eight backcross generations to overcome the confounding effects of background heterozygosity. The method used here enables chromosomal effects to be identified in two or three generations and thus represents a considerable saving in time and effort.

Acknowledgement AJSC is grateful to Imperial Chemical Industries PLC for a Research Studentship. 


\section{REFERENCES}

BINGHAM, J. 1967. Investigations of the physiology of yield in winter wheat, by comparis on of varieties and by artificial variation in grain number per ear. J. Agric. Sci., 68, 411422.

variation in grain number per ear. J. Agric. Sci., 68, 411-422.

BREMNER, P. M. AND RAWSON, H. M. 1978. The weights of individual grains of the wheat ear in relation to their growth potential, the supply of assimilate and interaction between grains. Aust. J. Plant Physiol., 5, 61-72.

BROCKLEHURST, P. A. 1977. Factors controlling grain weight in wheat. Nature, 266, 348-349.

JENNER, C. F. 1980. Effects of shading or removing spikelets in wheat: testing assumptions. Aust. J. Plant Physiol., 7, $113-122$.

KUSPIRA, J. AND UNRAU, J. 1957. Genetic analysis of certain characters in wheat using whole chromosome substitution lines. Can. J. Plant Sci., 37, 300-326.

KUSPIRA, J. AND UNRAU, J. 1959. Theoretical ratios and tables to facilitate genetic studies with aneuploids. I. $F_{1}$ and $F_{2}$ analysis. Can. J. Genetics and Cytology, 1, 267-312.

LAW, C. N. 1967. The location of genetic factors controlling a number of quantitative characters in wheat. Genetics, 56, 445-461.
LAW, C. N. AND wORLAND, A. J. 1973. Aneuploidy in wheat and its uses in genetic analysis. In Plant Breeding Institute Annual Report, 1972, Cambridge, 25-65.

LAW, C. N., SNAPE, J. W. AND WORLAND, A. J. 1979. Reciprocal monosomic studies. In Plant Breeding Institute Annual Report, 1978, Cambridge, 134-135.

LAW, C. N., SNAPE, J. W. AND WORLAND, A. J. 1981. Intraspecific chromosome manipulation. Phil. Trans. R. Soc. London B, 292, 509-518.

LUPTON, F. G. H. AND KIRBY, E. J. M. 1968. Applications of physiological analysis to cereal breeding. In Plant Breeding Institute Annual Report, 1967, Cambridge, 5-26.

MCEWAN, J. M. AND KALTSIKES, P. J. 1970. Early generation testing as a means of predicting the value of specific chromosome substitutions into common wheat. Can. J. Genet. Cytol., 12, 711-723.

SEARS, E. R. 1953. Nullisomic analysis in common wheat. Amer. Nat., 87, 245-252.

SEARS, E. R. 1954. The aneuploids of common wheat. Mo. Agric. Exp. Stn. Res. Bull. 572.

SIMMONS, S. R. AND MOSS, D. N. 1978. Nitrogen and dry matter accumulation by kernels formed at specific florets in spikelets of spring wheat. Crop. Sci., 18, 139-143.

WARDLAW, I. F. AND MONCUR, L. 1976. Source, sink and hormonal control of translocation in wheat (cv Gabo). Planta, 128, 93-100. 